\title{
Cancer in older adults: understanding cause and effects of chemotherapy-related toxicities
}

\author{
Vijaya R Bhatt*,1,2 \\ ${ }^{1}$ Department of Internal Medicine, Division of Hematology-Oncology, University of Nebraska Medical Center, Omaha, NE 68198, \\ USA \\ ${ }^{2}$ Fred \& Pamela Buffett Cancer Center, University of Nebraska Medical Center, Omaha, NE 68198, USA \\ *Author for correspondence: Tel.. +1 402559 5520; Fax: +1 402559 6520; vijaya.bhatt@unmc.edu
}

\section{"Reducing the risks and consequences of chemotherapy-related toxicities will evidently require expanding our knowledge base."}

First draft submitted: 22 March 2019; Accepted for publication: 1 May 2019; Published online: 24 July 2019

Keywords: cancer treatment $\bullet$ older adults $\bullet$ toxicities

In an aging population, the risk of cancer in older adults aged 60 years or older is expected to rise significantly over the next decade. Older adults are more likely to develop and die from various hematological and solid cancers. Cancer-related complications, chemoresistance resulting in refractory cancer and cancer relapses certainly contribute to high mortality in older adults. For example, older adults with acute myeloid leukemia (AML) have a greater likelihood of high-risk karyotype and mutations resulting in a significantly lower probability of achieving remission [1,2]. Appropriately, the field of cancer research has maintained a focus on understanding cancer biology to enhance drug development, which has resulted in an unprecedented approval of new drugs for cancer in the last few years. In the last 2 years, eight new drugs have received approval from the US FDA for management of AML [1]. Supportive care such as use of antimicrobial agents, transfusion and growth factors have made chemotherapy safer for patients in general. However, in older adults, chemotherapy intolerance and toxicities play a larger role in poor survival and quality of life than what may have been appreciated by the oncology community in the past. For this reason, in recent years, patient-reported outcomes, geriatric assessment and symptom science have been increasingly incorporated in cancer care and research.

\section{Why are older adults with cancer at a higher risk of chemotherapy-related toxicities?}

Older adults, compared with younger adults, are at a multiple-fold higher risk of developing toxicities, more so with intensive chemotherapy. Older adults with cancer often have multiple comorbidities, poor functional status, low organ function and poor hematopoietic potential [1,2]. Such frailty phenotype may increase the risk of chemotherapy-related toxicities (Figure 1) [3,4]. Therapy-related or secondary malignancies are also more common in older patients [5,6]. Race or ethnicity, gender, income status, education or health literacy, social support and rural location may modulate the risk of toxicities because of access to care including supportive care interventions, difficulties with adherence to medical recommendations, and possibly biological reasons in case of race and gender [7]. We have previously demonstrated higher risk of early mortality and overall survival based on socioeconomic and health system factors [8,9]. Clinical trials often have stringent eligibility criteria and exclude older adults, especially those with inadequate organ function and multimorbidity [10]. Consequently, enrollment in clinical trials is poor, particularly for older adults. Hence, appropriate therapy and drug doses may not be clear for older adults who are treated in real-world clinical practices. Finally, a higher probability of chemoresistance means a higher need for additional chemotherapy or reinduction in case of acute leukemia, and higher risk of toxicities. 


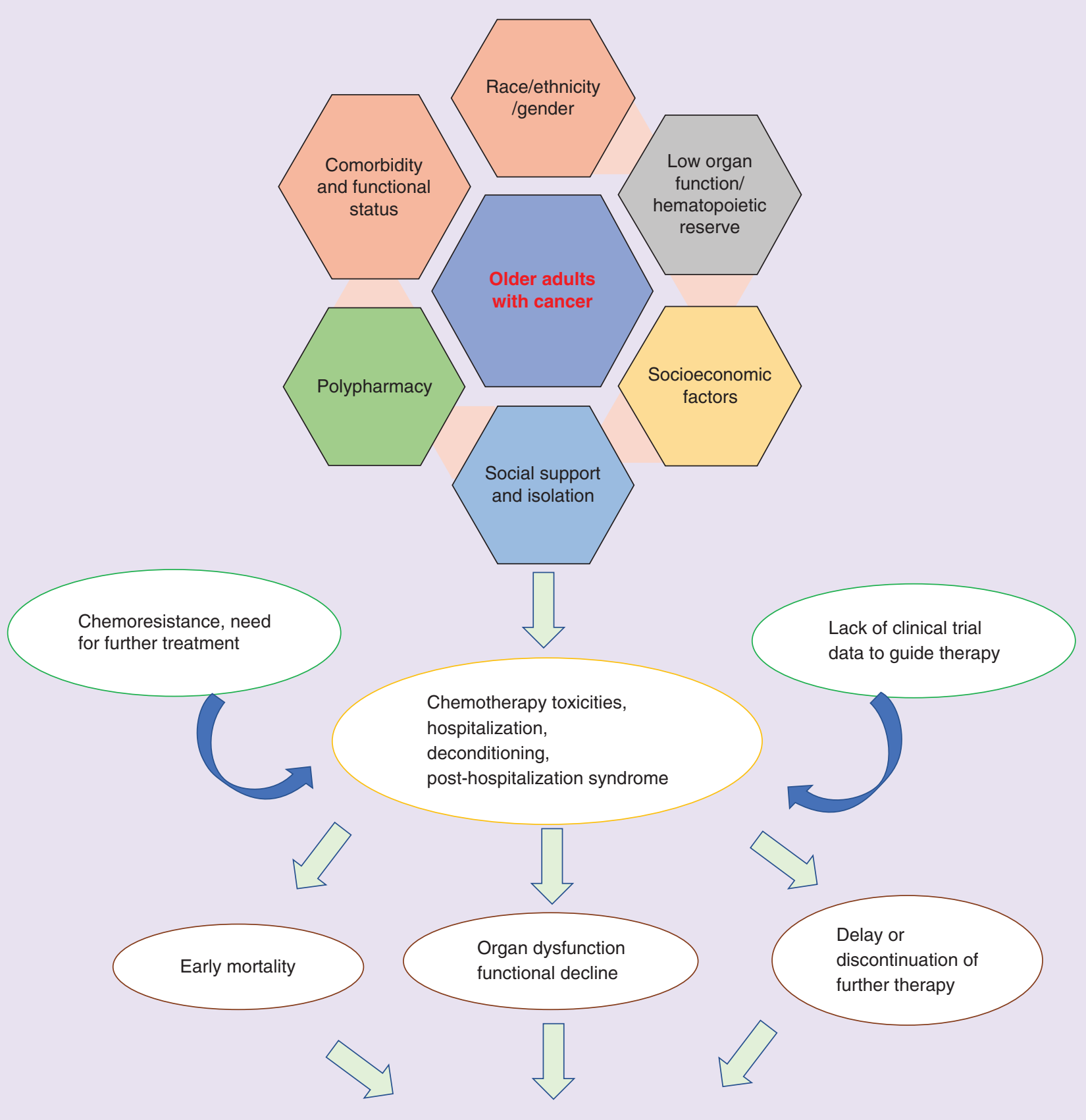

Poor long-term survival

Figure 1. A theoretical framework to explain the causal mechanisms of toxicities and its effect on survival of older adults.

\section{What are the predictors of chemotherapy-related toxicities?}

Increasing age, declining performance status and increasing comorbidity burden are common predictors of toxicities. Scoring systems have been developed to determine the risk of toxicities and early mortality. In AML, for instance, patient characteristics and laboratory tests have been incorporated to develop models that predict early death [11,12]. In the last decade, multiple large-scale studies have demonstrated geriatric assessment to predict chemotherapyrelated toxicities in solid cancers, and more recently in hematological cancers as well [3,4]. Many of these tools 
are available online for public use. One example is a chemotherapy toxicity calculator, developed by the Cancer and Aging Research Group based on a multicenter study [3]. This tool is available at the following weblink: www.mycarg.org/Chemo_Toxicity_Calculator. These tools can be utilized to predict risk of toxicities and involve patients in shared decision making regarding the use of varying intensity of chemotherapy.

\section{What are the consequences of developing chemotherapy-related toxicities?}

Serious adverse events can be life threatening and can result in early mortality, or permanent organ dysfunction such as kidney damage. Patients may require frequent emergency care or hospitalization and develop posthospital syndrome [13]. Posthospital syndrome refers to a state of vulnerability noted after a recent hospitalization, when patients are at an increased risk of several new medical problems [13]. Toxicities and recurrent hospitalization can result in functional decline, and poor quality of life. Such consequences may delay or result in reduction or discontinuation of further chemotherapy and transplant. Toxicities, dose delays, reduction and discontinuation of chemotherapy may result in poor long-term survival. Concerns for toxicities may affect physicians' recommendations and patients' preferences to utilize chemotherapy or hematopoietic cell transplant and enroll in clinical trials. Multiple studies have demonstrated that many older adults do not receive chemotherapy for a new diagnosis of cancer or hematopoietic cell transplant $[8,9,14,15]$. In a large National Cancer Database study, increasing age was associated with a lower rate of chemotherapy utilization for initial management of AML. Approximately one-third of older adults aged 71-80 years and two-thirds of those aged $\geq 81$ years did not receive any chemotherapy [9,15]. Among adults aged 61-75 years with intermediate to high-risk AML, only $5.5 \%$ of patients underwent a hematopoietic cell transplant [14]. Rates of clinical trial participation are very low at 3-5\% among adults in general in the USA. An analysis by the FDA demonstrated that older adults are disproportionately under-represented in clinical trials despite older adults constituting a high proportion of the US cancer population [16]. While multiple factors likely explain these observations, concerns for toxicities play a significant role. At broader levels, chemotherapy-related toxicities can add to caregiver burden, financial toxicity and healthcare cost. Caregiver burden is an important issue as caregivers of older adults are frequently of similar age and suffer from multiple health problems.

\section{Are there any potential solutions?}

Older adults at high risk of complications should ideally be treated by a multidisciplinary team comprising of oncologists, geriatricians, physical therapists, dieticians, pharmacists and other specialists [2]. Guidelines from geriatric oncology groups provide recommendations on supportive care interventions for each of the health impairments identified by a geriatric assessment $[17,18]$. Such interventions may include physical or occupational therapy, home safety evaluation, fall and delirium precautions, nutritional support, psychotherapy and drugs to treat depression. These interventions may reduce the risk of toxicities and functional decline. Ongoing studies are evaluating the effect of such supportive care interventions and prehabilitation programs in older adults with cancers. Educating patients' about potential risks and benefits of various treatments and understanding patients' preference may help in selecting therapy that meets patients' desired balance between safety and efficacy of treatment. We have recently developed a self-report questionnaire, 'therapy preference scale' [19], which we hope will provide an easy tool to gain a deeper understanding of patients preferences and guide shared decision making.

Reducing the risks and consequences of chemotherapy-related toxicities will evidently require expanding our knowledge base. Expansion of eligibility criteria and development of trials specifically for older adults may increase enrollment of older adults in clinical trials and provide data to guide therapy in real world [10]. In some situation, pharmacokinetic, pharmacodynamic and dose-finding studies may be necessary specifically in older adults before a drug can be routinely used in this population. Molecular biology and physiological research to understand mechanisms that result in specific toxicities may allow development of targeted drugs or other specific prevention strategies. Clinical trials may need to be designed to personalize therapy selection in older adults based on anticipated risk of toxicities and benefits of intensive chemotherapy [1]. At our center, we currently utilize geriatric assessment and genetic test results to personalize selection of intensity of chemotherapy as a part of an ongoing clinical trial for older adults with AML (ClinicalTrials.gov: NCT03226418). Whereas geriatric assessment can predict chemotherapy-related toxicities, cytogenetic risk categories correlate with the probability of remission achieved with intensive chemotherapy [1].

While clinical trials routinely capture and report data on toxicities, toxicities have not been evaluated as predictors of survival. Large-scale studies are necessary to quantify the effect of serious adverse events and grade $\geq 3$ toxicities 
on survival of older adults. Such studies will highlight the importance of preventing chemotherapy-related toxicities in older adults and provide strong rationale for promoting further investigation in this field.

\section{Author contributions}

VR Bhatt wrote the manuscript.

\section{Financial \& competing interests disclosure}

This work was supported by the National Institute of General Medical Sciences, 1 U54 GM115458, which funds the Great Plains Institutional Development Award (IDeA) Clinical Translational Research (CTR) Network, and the Fred and Pamela Buffett Cancer Center Support Grant from the National Cancer Institute (P30 CA036727). The content is solely the responsibility of the author and does not necessarily represent the official views of the NIH. VR Bhatt reports receiving consulting fees from Pfizer, CSL Behring, Agios, Incyte, Partner Therapeutics and Abbvie, and research funding from Incyte, Tolero Pharmaceuticals, Inc, and National Marrow Donor Program. The author has no other relevant affiliations or financial involvement with any organization or entity with a financial interest in or financial conflict with the subject matter or materials discussed in the manuscript apart from those disclosed.

No writing assistance was utilized in the production of this manuscript.

\section{References}

1. Bhatt VR. Personalizing therapy for older adults with acute myeloid leukemia: role of geriatric assessment and genetic profiling. Cancer Treat. Rev. 75(May), 52-61 (2019).

2. Bhatt VR, Gundabolu K, Koll T, Maness LJ. Initial therapy for acute myeloid leukemia in older patients: principles of care. Leuk. Lymphoma 59(1), 29-41 (2018).

3. Hurria A, Togawa K, Mohile SG et al. Predicting chemotherapy toxicity in older adults with cancer: a prospective multicenter study. $J$. Clin. Oncol. 29(25), 3457-3465 (2011).

4. Hamaker ME, Prins MC, Stauder R. The relevance of a geriatric assessment for elderly patients with a haematological malignancy - a systematic review. Leuk. Res. 38(3), 275-283 (2014).

5. Bhatt VR, Giri S, Verma V et al. Secondary acute myeloid leukemia in survivors of Hodgkin lymphoma. Future Oncol. 12(13), 1565-1575 (2016).

6. Bhatt VR. Leukemic transformation in essential thrombocythemia. Future Oncol. 10(16), 2593-2602 (2014).

7. Kirtane K, Lee SJ. Racial and ethnic disparities in hematologic malignancies. Blood 130(15), 1699-1705 (2017).

8. Bhatt VR, Shostrom V, Giri S et al. Early mortality and overall survival of acute myeloid leukemia based on facility type. Am. J. Hematol. 92(8), 764-771 (2017).

9. Bhatt VR, Shostrom V, Armitage JO, Gundabolu K. Treatment patterns and outcomes of octogenarian patients with acute myeloid leukemia. Am. J. Hematol. 94(6), e169-e172 (2019).

10. Hurria A, Levit LA, Dale W et al. Improving the evidence base for treating older adults with cancer: American Society of Clinical Oncology statement. J. Clin. Oncol. 33(32), 3826-3833 (2015).

11. Krug U, Rollig C, Koschmieder A et al. Complete remission and early death after intensive chemotherapy in patients aged 60 years or older with acute myeloid leukaemia: a web-based application for prediction of outcomes. Lancet 376(9757), 2000-2008 (2010).

12. Walter RB, Othus M, Borthakur G et al. Prediction of early death after induction therapy for newly diagnosed acute myeloid leukemia with pretreatment risk scores: a novel paradigm for treatment assignment. J. Clin. Oncol. 29(33), 4417-4423 (2011).

13. Krumholz HM. Post-hospital syndrome - an acquired, transient condition of generalized risk. N. Engl. J. Med. 368(2), 100-102 (2013).

14. Bhatt VR, Chen B, Gyawali B, Lee SJ. Socioeconomic and health system factors associated with lower utilization of hematopoietic cell transplantation in older patients with acute myeloid leukemia. Bone Marrow Transplant. 53(10), 1288-1294 (2018).

15. Bhatt VR, Shostrom V, Gundabolu K, Armitage JO. Utilization of initial chemotherapy for newly diagnosed acute myeloid leukemia in the United States. Blood Adv. 2(11), 1277-1282 (2018).

16. Talarico L, Chen G, Pazdur R. Enrollment of elderly patients in clinical trials for cancer drug registration: a 7-year experience by the US Food and Drug Administration. J. Clin. Oncol. 22(22), 4626-4631 (2004).

17. Mohile SG, Dale W, Somerfield MR et al. Practical assessment and management of vulnerabilities in older patients receiving chemotherapy: ASCO Guideline for Geriatric Oncology. J. Clin. Oncol. 36(22), 2326-2347 (2018).

18. Mohile SG, Velarde C, Hurria A et al. Geriatric assessment-guided care processes for older adults: a Delphi consensus of geriatric oncology experts. J. Natl Compr. Canc. Netw. 13(9), 1120-1130 (2015).

19. Bhatt VR. Understanding patients' values and priorities in selecting cancer treatments: developing a therapy preference scale. J. Geriatr. Oncol. doi:10.1016/j.jgo.2018.11.005 (2018) (Epub ahead of print). 